\title{
WHIPPLE'S DISEASE MIMICKING UNDIFFERENTIATED ARTHRITIS AND FIBROMYALGIA
}

Raíssa Dudienas Domingues Pereira ${ }^{1, \star}$, Diego de Paula Ferreira Nunes ${ }^{1}$, Vinícius Verlangieri Soubihe ${ }^{1}$, Caio Rustichelli Cardoso ${ }^{1}$, Alisson Pugliesi ${ }^{1}$, Juliana Zonzini Gaino ${ }^{1}$, Michel Alexandre Yazbek $^{1}$, Lilian Tiemi Hirata ${ }^{1}$, Manoel Barros Bertolo ${ }^{1}$

1.Universidade Estadual de Campinas, Campinas (SP), Brazil.

*Corresponding author: ra_dudienas@hotmail.com

\section{BACKGROUND}

Whipple disease is a rare systemic disease caused by a gram-positive intracellular bacterium, (Tropheryma whipplei) with an estimated prevalence of one in a million and with a preference to affect middle-aged white men. It is characterized by nonspecific gastrointestinal symptoms, which are usually preceded by joint symptoms by many years.

\section{CASE REPORT}

Female, 44 years old, caucasian, no comorbidities. She started rheumatologic follow-up in 2013 due to migratory polyarthritis, with associated morning stiffness lasting approximately two hours and partial and temporary improvement with the use of prednisone. On physical examination, hyperemia, increased temperature and pain on palpation of the left wrist and left medial epicondyle. Laboratory tests showed an increase in inflammatory tests and thrombocytosis. All serologies were negative as was rheumatoid factor, and the X-rays showed no alterations. The diagnosis of an undifferentiated arthritis was done and prednisone $5 \mathrm{mg}$ and hydroxychloroquine $400 \mathrm{mg}$ were started. On subsequent visits, there was no more evidence of arthritis. The patient complained of nonrestorative sleep and presented pain on palpation of tender points, in addition to myofascial pain and the diagnosis of undifferentiated arthritis was changed to fibromyalgia. After six years of follow-up, the patient comes to the consultation with a report of loose stools for one year and weight loss of $20 \mathrm{~kg}$ in 6 months, having started follow-up with a gastroenterologist. In view of the situation, a new diagnostic hypothesis was suggested: enteropathic spondyloarthritis. A colonoscopy was done with no specific findings (ileitis and chronic rectocolitis); a magnetic resonance imaging of the abdomen was also required, which showed retroperitoneal lymph node enlargement, with the largest in the flank, measuring $4.5 \times 2.2 \mathrm{~cm}$. Given the findings of lymph node enlargement, it was decided to undergo a retroperitoneal lymph node biopsy, which pathological examination showed chronic duodenitis with enlargement of the villi due to the presence of numerous macrophages filled with PAS positive material, in addition to dilated lymphatics and fat vacuoles, compatible with Whipple's disease. Treatment was then started with IV ceftriaxone for 14 days and $\mathrm{VO}$ amoxicillin + clavulanate for a long time, with the patient presenting improvement in the diarrheal condition and complete resolution of the rheumatology complaints.

\section{CONCLUSION}

Whipple's disease is rare and difficult to diagnose, and its signs and symptoms can mimic several rheumatologic diseases, delaying diagnosis. The rheumatologist should remember this entity in cases of refractory disease, especially when there are also Gl symptoms.

\section{KEYWORDS}

Whipple's disease, Fibromyalgia, Undifferentiated arthritis. 